\title{
Lack of clastogenic effects of L-thyroxine in whole-blood cultured human lymphocytes
}

\author{
Ninoslav Djelic ${ }^{1}$, Dijana Djelic ${ }^{2 \dagger}$, Biljana Spremo-Potparevic ${ }^{3}$, Lada Zivkovic ${ }^{3}$, Biljana Markovic ${ }^{1}$, \\ Olivera Lozance $^{2}$ and Milos Blagojevic ${ }^{2}$ \\ ${ }^{1}$ Department of Biology, Faculty of Veterinary Medicine, University of Belgrade, Belgrade, Serbia. \\ ${ }^{2}$ Department of Anatomy, Faculty of Veterinary Medicine, University of Belgrade, Belgrade, Serbia. \\ ${ }^{3}$ Department of Biology and Human Genetics, Institute of Physiology, Faculty of Pharmacy, \\ University of Belgrade, Belgrade, Serbia.
}

\begin{abstract}
Thyroid hormones stimulate aerobic metabolism which may lead to oxidative stress accompanied by damage to various cellular macromolecules, including DNA. Previous comet assay studies have shown that thyroid hormones cause DNA damage due to the creation of reactive oxygen species (ROS). However, cytogenetic studies have been equivocal because although an increase in the sister-chromatid exchange frequency per cell has been reported increased micronuclei frequency has not. We used cytogenetic examination of chromosome breakage and aberrations in whole-blood cultures of human peripheral blood lymphocytes to investigate possible clastogenic effects when lymphocytes were exposed to $0.002 \mu \mathrm{M}$ to $50 \mu \mathrm{M}$ of L-thyroxine for $24 \mathrm{~h}$ and $48 \mathrm{~h}$, these concentrations being chosen because they had been used in previous studies of sister-chromatid exchange and micronuclei frequency. Under our experimental conditions thyroxine did not induced any statistically significant increase in chromosome breakage or aberrations. This lack of clastogenic effects is in contrast to the reported comet assay results obtained using purified lymphocytes, possibly because whole-blood cultures contain catalase and glutathione peroxidase capable of reducing the effects of reactive oxygen species.
\end{abstract}

Key words: thyroxine, human lymphocytes, chromosome aberrations, mitotic index, ROS.

Received: November 6, 2006; Accepted: May 17, 2007.

Thyroid hormones stimulate development, growth and metabolism in most vertebrate tissues (Lazar, 2003). At the molecular level, increased metabolic rate and oxygen consumption is achieved both by the short-term action of 3,5-didodothyronine on mitochondrial cytochrome oxidase $c$ and long-term changes of nuclear and mitochondrial gene transcription mediated by 3,3',5-triiodothyronine (Goglia et al., 1999). The molecular mechanisms of thyroid hormone action have been thoroughly investigated but data is still sparse on possible genotoxic and mutagenic effects. Although it has been shown that thyroxine $\left(\mathrm{T}_{4}\right)$ acts as a co-carcinogen in the development of rat colon cancer (Iishi et al., 1992) and that prolonged administration of thyroxine increases gastric carcinogenesis induced by $N$-methyl- $N^{*}$-nitro- $N$-nitrosoguanidine in Wistar rats (Iishi et al., 1993), these effects resulted from non-genotoxic

Send correspondence to Ninoslav Djelic. University of Belgrade, Faculty of Veterinary Medicine, Department of Biology, Bul. Oslobodjenja 18, 11000 Belgrade, Serbia E-mail: ndjelic@vet.bg. ac.yu.

${ }^{\dagger}$ Deceased. mechanisms possibly due to the stimulation of cell proliferation. It is known, however, that thyroid hormones increase aerobic metabolism in mitochondria and causes intense production of reactive oxygen and nitrogen species, leading to the condition of oxidative stress (Venditti et al., 2003; Fernandez et al., 2005). Moreover, binding of thyroid hormones to specific nuclear receptors in target cells induces expression of enzymes related to redox processes and the total rate of oxygen consumption is enhanced (Oppenheimer et al., 1996). Oxidative stress induced by thyroid hormones can lead to lipid and protein oxidation in rat liver cells (Tapia et al., 1999). Experimentally induced hyperthyroidism in rat liver is accompanied by an increased rate of hepatic $\mathrm{O}_{2}$ consumption, enhanced microsomal oxidative capacity and the generation of reactive oxygen species (ROS) (Fernandez et al., 2003). In addition, treatment with thyroid hormone decreases the activity of superoxide dismutase (SOD), catalase and glutathione content in rat liver (Fernandez et al., 1988). This depression of key antioxidative mechanisms favors oxidative stress in the liver (Tapia et al., 1999). 
It is well established that oxidative stress causes damage to biological molecules such as proteins, membrane lipids and DNA. Oxidative DNA damage can produce a wide variety of DNA modifications including base and sugar lesions, strand breaks, DNA-protein cross-links and base-free sites (Evans et al., 2004). Elevated levels of thyroid hormones can enhance production of ROS in mitochondria not only in organs under the strong influence of thyroid status (i.e. the liver), but also in human leukocytes (Magsino et al., 2000). Indeed, more recent experimental findings have shown that thyroid hormones induce DNA damage in human lymphocytes (Djelic and Anderson, 2003) and sperm (Dobrzynska et al., 2004), which can be observed in the comet assay. Since the antioxidant enzyme catalase reduced these effects, DNA damage was caused mainly by action of ROS. In our previous investigations, we also studied the cytogenetic effects of L-thyroxine on human peripheral blood lymphocytes by monitoring sister-chromatid exchange (SCE) and micronuclei as endpoints of the genotoxic exposure (Djelic et al., 2006). However, thyroxine exhibited only weak genotoxic effects expressed as an increased frequency of SCE per cell in cultured human lymphocytes, whereas no effect was observed in the cytokinesis block micronucleus assay (Djelic et al., 2006). Since these results of cytogenetic evaluation of thyroxine toxicity were equivocal, we undertook the investigation described in this paper to further elucidate possible clastogenic effects of thyroxine on cultured human lymphocytes.

The thyroid hormone L-thyroxine (3-[4-(4-Hydroxy3,5-diiodophenoxy)-3,5-diiodophenyl]-1-alanine (or $\mathrm{T}_{4}$ ), molecular mass 776.87, CAS No. 51-48-9) was investigated at concentrations ranging from $0.002 \mu \mathrm{M}$ to $50 \mu \mathrm{M}$ (Table 1) dissolved in dimethyl sulfoxide (DMSO). In addition to untreated cultures, the negative control was $0.8 \%$ (v/v) DMSO (final conc. $0.1 \mathrm{M}$ ), without thyroxine, and the positive control was the mutagen $N$-methyl- $N^{6}$-nitro- $N$ nitrosoguanidine (MNNG, CAS No. 70-25-7), without DMSO, at a concentration of $1 \mu \mathrm{M}$. These chemicals were purchased from the Sigma Chemical Co., St. Louis, USA.

Human peripheral blood lymphocyte cultures were set up, as previously reported by Djelic et al. (1996), according to a slight modification of the protocol described by Evans and O'Riordan (1975). Briefly, $0.8 \mathrm{~mL}$ heparinized whole blood samples, obtained by venipuncture from three healthy Caucasian male volunteers under 30 years of age, were added to vials with $9.2 \mathrm{~mL}$ of RPMI 1640 media (Gibco, Grand Island, NY) supplemented with $15 \%(\mathrm{v} / \mathrm{v})$ of heat-inactivated fetal calf serum (Gibco, Eggenstein, Germany) and supplemented with 1\% (w/v) penicillin, $1 \%(\mathrm{w} / \mathrm{v})$ streptomycin (Galenika, Belgrade, Serbia) and $5 \mu \mathrm{g} \mathrm{mL}^{-1}$ of phytohemagglutinin (Murex Diagnostics Ltd., Dartford, England). Duplicate cultures from each donor were incubated in the dark for $72 \mathrm{~h}$ at $37^{\circ} \mathrm{C}$. The study was approved by the local Medical Ethics Commit- tee, performed in accordance with the Declaration of Helsinki, and informed donor consent was also obtained.

Two L-thyroxine exposure times were used ( $24 \mathrm{~h}$ and $48 \mathrm{~h})$, appropriate concentrations $(0.002 \mu \mathrm{M}$ to $50 \mu \mathrm{M}$, Table 1) of L-thyroxine dissolved in DMSO being added to the culture vials after $24 \mathrm{~h}$ incubation for the $48 \mathrm{~h}$ exposure experiment and at $48 \mathrm{~h}$ for the $24 \mathrm{~h}$ exposure experiment.

Two hours before harvesting $0.5 \mu \mathrm{g} \mathrm{mL}^{-1}$ colcemid (Ciba, Basel, Switzerland) was added to the cultures and the cells treated with hypotonic $0.075 \mathrm{M} \mathrm{KCl}$. After three standard cycles of fixation in methanol/acetic acid (3:1, $\mathrm{v} / \mathrm{v}$ ) the cell suspension was dropped onto chilled greasefree coded microscopic slides, air dried and stained in 5\% (v/v) Giemsa (Merck, Darmstadt, Germany). We scored 200 mitotic plates per donor (100 per each duplicate culture) for each experimental concentration and for the positive and negative controls. Scoring of gaps and breaks was performed according to Brogger (1982) and the mitotic index (MI) was calculated as the percentage of cells in mitosis based on at least 2000 cells per donor.

Statistical analysis of cytogenetic endpoints and mitotic index was carried out using the chi-squared $\left(\chi^{2}\right)$ test (Sivikova et al., 1999) at a significance level of $\mathrm{p}=0.05$ or lower.

In addition to the cytogenetic analysis we monitored possible cytotoxic or mitogenic effects of thyroxine using the analysis of the mitotic index on the same slides, the results for both types of analysis being shown in Table 1 .

To evaluate the possible clastogenic effects of L-thyroxine we analyzed cultured whole-blood lymphocytes for chromosome lesions (gaps and breaks), rearrangements and numeric and structural aberrations, but we found no statistically significant structural chromosome aberrations after treatment with L-thyroxine dissolved in DMSO when compared to the negative control lymphocytes exposed to DMSO only, nor in comparison to untreated cultures, even though various types of chromosome lesions were detected and carefully scored by three independent assessors (Table 1). Thus, the overall percentage of aberrant cells in L-thyroxine treated cultures was in the range of $0 \%$ to $4.5 \%$ and there was no significant departure $(p>0.05)$ form the negative control base level. This agrees with our previous investigations (Djelic et al., 2006) in which we observed no significant effect in the cytokinesis block micronucleus assay, using blood samples from the same donors and the same assay conditions. As expected, the positive control (MNNG) caused significant clastogenic effects, detectable as an increase in breaks and chromatid and isochromatid exchanges $(p<0.001)$, indicating that the experimental conditions were suitable for detecting clastogenic effects.

After $24 \mathrm{~h}$ exposure the mean mitotic index was 5.5\% for the negative control lymphocytes from all three donors. The experimental mitotic index values for cultures exposed to L-thyroxine for $24 \mathrm{~h}$ ranged from $2.4 \%$ to $6.1 \%$. The highest concentration $(50 \mu \mathrm{M})$ of L-thyroxine caused a sig- 


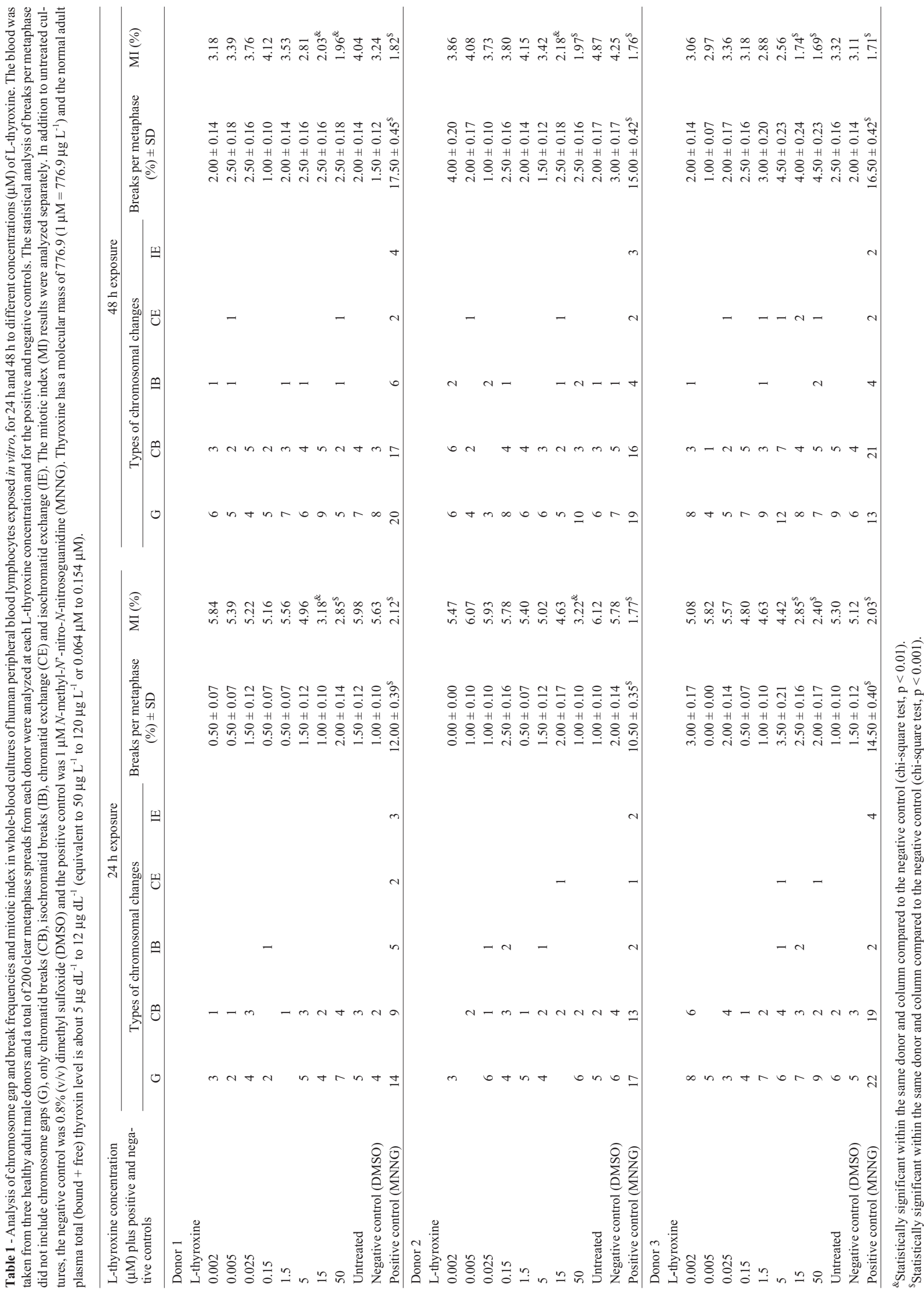


nificant decrease in mitotic index compared to the value of the negative control. Furthermore, at concentrations of $15 \mu \mathrm{M}$ the mitotic index was still significantly decreased for the lymphocytes from donor $1(\mathrm{p}<0.01)$ and donor 3 $(\mathrm{p}<0.001)$, whereas for donor 2 although there was a decrease in the mitotic index at this concentration the decrease was not significant. After $48 \mathrm{~h}$ exposure the mean mitotic index for the negative control lymphocytes from the three donors was $3.5 \%$, whereas the overall range in cultures treated with L-thyroxine was from $1.7 \%$ to $4.1 \%$. The two highest L-thyroxine concentrations (15 $\mu \mathrm{M}$ and $50 \mu \mathrm{M}$ ) caused a significant decrease of mitotic index in the lymphocytes from all three donors at $48 \mathrm{~h}$ (Table 1). However, these concentrations far exceed not only the normal blood thyroxine level $(\sim 0.1 \mu \mathrm{M})$ and level in L-thyroxine treated patients, but also L-thyroxine level both in severe hyperthyroidism (Ganong, 1995) and acute L-thyroxine overdose (Berkner et al., 1991). As expected, the positive control significantly lowered the mitotic index values for the lymphocytes from all three donors, both after $24 \mathrm{~h}$ and $48 \mathrm{~h}$ of exposure (Tables 1 ).

Investigations in the field of genetic toxicology have mainly concentrated on environmental pollutants, most of which result from intense development of chemical and other industries (Pitarque et al., 2002; Stanimirovic et al., 2005). However, there is increasing evidence that substances which are normally present in human or animal bodies may, under the certain circumstances, exhibit deleterious effects on genetic material and therefore act as endogenous mutagenic agents (Lutz, 1990; Totsuka et al, 2005).

One of the best studied group of endogenous mutagenic agents are estrogenic hormones (Anderson et al., 2003; Schallreuter et al., 2006). It is well established that metabolic conversion of phenolic estrogen moieties accompanied by the generation of ROS leads to covalent damage of cellular macromolecules including DNA (Liehr, 2001; Li et al., 2004). Interestingly, there are some experimental indications that phenolic groups of some non-steroid hormones (adrenaline, thyroid hormones) and neurotransmitters (dopamine, noradrenaline) may undergo redox cycling accompanied by the generation of ROS which favor oxidative stress (Moldeus et al., 1983; Djelic and Anderson, 2003; Dobrzynska et al., 2004). It thus seems that oxidative stress is the key mechanism of the genotoxic effects of both steroid and non-steroid hormones with phenolic groups.

The comet assay has shown that thyroid hormones can cause in vitro DNA damage to purified human lymphocytes (Djelic and Anderson, 2003) and sperm (Dobrzynska et al, 2004) and that the damage can be reduced by the antioxidant enzyme catalase, indicating that the genotoxic effects of thyroid hormones may be principally due to ROS. The suggestion that L-thyroxine can have genotoxic effects is supported by reports of increased SCE in human lymphocytes treated with relatively high concentrations of L-thy- roxine, although since L-thyroxine did not induce micronuclei under the same experimental conditions the cytogenetic effects of L-thyroxine were unclear (Djelic et al., 2006). It is important to note, however, that in the study by Djelic and Anderson (2003) the DNA damage attributed to thyroid hormones was detected by the comet assay of purified human lymphocytes, with the cytogenetic evaluation of the genotoxic effects of L-thyroxine on lymphocytes from whole blood cultures producing equivocal results. These discrepancies may be explained, at least in part, by the findings of Andreoli et al. (1999) who reported more severe genotoxic effects on purified lymphocytes than compared to lymphocytes from whole-blood cultures, probably because erythrocytes in whole blood contain catalase and glutathione peroxidase which eliminate hydrogen peroxide and reduce the amount of ROS available for generating DNA damage.

Our results show that L-thyroxine did not produce clastogenic effects which could be detected on the basis of cytogenetic analysis and supports previous observations that L-thyroxine does not cause any significant increase in the micronucleus frequency of human peripheral blood lymphocytes (Djelic et al., 2006). However, it should be pointed out that thyroid hormones caused DNA damage in the comet assay, at high concentrations in a range 10-100 $\mu \mathrm{M}$ (Djelic and Anderson, 2003; Dobrzynska et al, 2004). Although these concentrations are comparable with two highest concentrations of L-thyroxine in the present study, genotoxic effects were observed only in the comet assay, which is more sensitive in comparison to the cytogenetic analysis.

Analysis of clastogenic effects has not revealed any significant difference between untreated cultures and negative controls treated with DMSO (Table 1), as observed previously by de Lima et al. (2005). Also, there was no significant response in thyroxine-treated cultures, in comparison to both untreated and DMSO treated cultures.

The percentage of cells in mitosis was significantly decreased only at two highest L-thyroxine concentrations, possibly due to cytotoxic effects. This finding is in accordance with our previous cytogenetic study (Djelic et al., 2006) in which we observed decreases in mitotic index at the same experimental concentrations of L-thyroxine. As expected, positive control caused a more profound decrease in mitotic index, possibly due to arrested mitosis as a result of the repair of genetic damage or cytotoxic effects due to relatively high levels of genetic damage.

The results indicate that normal levels of L-thyroxine do not produce clastogenic effects on human lymphocytes in whole-blood cultures. In this investigation, the concentration of L-thyroxine of $0.15 \mu \mathrm{M}$ corresponds to the total plasma level in euthyroid persons (Ganong, 1995). The higher concentration of $1.5 \mu \mathrm{M}$ exceeds the total serum L-thyroxine concentration in Graves disease, but it is comparable to L-thyroxine blood level in an acute overdose 
(Berkner et al., 1991). Although L-thyroxine can increase the SCE rate (Djelic et al. 2006), the molecular mechanisms of SCE formation and their biological significance are not clear. Furthermore, the SCE test only indicates exposure to genotoxins, while the chromosome aberration and micronucleus assays are mutagenicity tests (Speit and Henderson, 2005) and are therefore of more significance than the SCE test. Based on these results we conclude that, under the experimental conditions employed in this study, L-thyroxine does not exhibit cytogenetically detectable genotoxic effects in lymphocytes from whole-blood cultures.

\section{Acknowledgments}

This investigation was supported by the Serbian Ministry of Science, grant \#143018B.

\section{References}

Anderson D, Schmid TE, Baumgartner A, Cemeli-Carratala E, Brinkworth M and Wood JM (2003) Oestrogenic compounds and oxidative stress (in human sperm and lymphocytes in the Comet assay). Mutat Res 544:173-178.

Andreoli C, Rossi S, Leopardi P and Crebelli R (1999) DNA damage by hydroquinone in human white blood cells: Analysis by alkaline single-cell gel electrophoresis. Mutat Res 438:37-45.

Berkner PD, Starkman H and Person N (1991) Acute thyroxine overdose; therapy with sodium ipodate: Evaluation of clinical and physiologic parameters. J Emerg Med 9:129-131.

Brogger A (1982) The chromatid gap: A useful parameter in genotoxicology? Cytogenet Cell Genet 33:14-19.

de Lima PD, Yamada ES, da Costa ET, Pessoa C do O, Rabenhorst SH, Bahia M de O, Cardoso PC, Santos RA, Smith M de A and Burbano RR (2005) Genotoxic effects of rotenone on cultured lymphocytes. Genet Mol Res 4:822831

Djelic N, Spremo-Potparevic B, Bajic V and Djelic D (2006) Sister chromatid exchange and micronuclei in human peripheral blood lymphocytes treated with thyroxine in vitro. Mutat Res 604:1-7.

Djelic N and Anderson D (2003) The effect of the antioxidant catalase on oestrogens, triiodothyronine and noradrenaline in the Comet assay. Teratog Carcinog Mutagen 23:69-81.

Djelic N, Soldatovic B, Andjelkovic M and Cvetkovic D (1996) In vitro cytogenetic analysis of the effects of oxytocin on human peripheral blood lymphocytes. Mutat Res 356:265-268.

Dobrzynska MM, Baumgartner A and Anderson D (2004) Antioxidants modulate thyroid hormone- and noradrenalineinduced DNA damage in human sperm. Mutagenesis 19:325-330.

Evans HJ and O'Riordan ML (1975) Human peripheral blood lymphocytes for the analysis of chromosome aberrations in mutagen tests. Mutat Res 31:135-148.

Evans MD, Dizdaroglu M and Cooke MS (2004) Oxidative DNA damage: Induction, repair and significance. Mutat Res 567:1-61.

Fernandez V, Llesuy S, Solari L, Kiperos K, Videla LA and Boveris A (1988) Chemiluminiscent and respiratory re- sponses related to thyroid hormone-induced liver oxidative stress. Free Rad Res Commun 5:77-84.

Fernandez V, Massa L, Quinones L, Simon-Giavarotti KA, D'Almeida V, Azzalis LA, Junqueira VB and Videla LA (2003) Effects of gamma-hexachlorocyclohexane and L3,3',5-triiodothyronine on rat liver cytochrome P450E21dependent activity and content in relation to microsomal superoxide radical generation. Biol Res 36:359-365.

Fernandez V, Tapia G, Varela P, Romanque P, Cartier-Ugarte D and Videla LA (2005) Thyroid hormone-induced oxidative stress in rodents and humans: A comparative view and relation to redox regulation of gene expression. Comp Biochem Physiol C Toxicol Pharmacol 142:231-239.

Ganong WF (1995) Review of Medical Physiology. Appleton and Lange, Sherwood, pp 306.

Goglia F, Moreno M and Lanni A (1999) Action of thyroid hormones at cellular level: The mitochondrial target. FEBS Lett 452:115-120.

Iishi H, Tatsuta M, Baba M, Okuda S and Taniguchi H (1992) Enhancement by thyroxine of experimental carcinogenesis induced in rat colon by azoxymethane. Int J Cancer 50:974976.

Iishi H, Tatsuta M, Baba M, Yamamoto R and Taniguchi H(1993) Enhancement by thyroxine of gastric carcinogenesis induced by N-methyl-N'-nitro-N-nitrosoguandine in Wistar rats. Br J Cancer 68:515-518.

Lazar MA (2003) Thyroid hormone action: A binding contract. J Clin Invest 112:497-499.

Li KM, Todoroviæ R, Deveanesan P, Higginbotham S, Kofeler H, Ramanathan R, Gross ML, Rogan EG and Cavalieri EL (2004) Metabolism and DNA binding studies of 4-hydroxyestradiol and estradiol-3,4-quinone in vitro and in female ACI rat mammary gland in vivo. Carcinogenesis 25:289297.

Liehr JG (2001) Genotoxicity of the steroidal oestrogens oestrone and oestradiol: Possible mechanism of uterine and mammary cancer development. Hum Reprod Update 7:273-281.

Lutz WK (1990) Endogenous genotoxic agents and processes as a basis of spontaneous carcinogenesis. Mutat Res 238:287295.

Magsino CH Jr, Hamouda W, Ghanim H, Browne R, Aljada A and Dandona P (2000) Effect of triiodothyronine on reactive oxygen species generation by leukocytes, indices of oxidative damage, and antioxidant reserve. Metabolism 49:799-803.

Moldeus P, Nordenskjold M, Bolcsfoldi G, Eiche A, Haglund U and Lambert B (1983) Genetic toxicity of dopamine. Mutat Res 124:9-24.

Oppenheimer JH, Schwartz HL and Strait KA (1996) The molecular basis of thyroid hormone action. In: Braverman LE and Utiger RD (eds) Werner and Ingbar's The Thyroid. A Fundamental and Clinical Text. $7^{\text {th }}$ ed. Lippincott-Raven, New York, chapter 9, pp 162-184.

Pitarque M, Vaglenov A, Nosko M, Pavlova S, Petkova V, Hirvonen A, Creus A, Norppa H and Marcos R (2002) Sister chromatid exchanges and micronuclei in peripheral lymphocytes of shoe factory workers exposed to solvents. Environ Health Perspect 110:399-404.

Schallreuter KU, Chiuchiarelli G, Cemeli E, Elwary SM, Gillbro JM, Spencer JD, Rokos H, Panske A, Chavan B, Wood JM, et al. (2006) Estrogens can contribute to hydrogen peroxide generation and quinone-mediated DNA damage in periph- 
eral blood lymphocytes from patients with vitiligo. J Invest Dermatol 126:1036-1042.

Sivikova K and Dianovsky J (1999) Genotoxic activity of the commercial herbicide containing bifenox in bovine peripheral lymphocytes. Mutat Res 439:129-135.

Speit G and Henderson L (2005) Review of the in vivo genotoxicity tests performed with styrene. Mutat Res 589:67-79.

Stanimirovic Z, Stevanovic J, Jovanovic S and Andjelkovic M (2005) Evaluation of genotoxic effects of Apitol ${ }^{\circledR}$ (cymiazole hydrochloride) in vitro by measurement of sister chromatid exchange. Mutat Res 588:152-157.
Tapia G, Cornejo P, Fernandez V and Videla LA (1999) Protein oxidation in thyroid hormone-induced liver oxidative stress: Relation to lipid peroxidation. Toxicol Lett 106:209-214.

Totsuka Y, Nishigaki R, Enomoto S, Takamura-Enya T, Masumura K, Nohmi T, Kawahara N, Sugimura T and Wakabayashi K (2005) Structures and biological properties of DNA adducts derived from N-nitroso bile acid conjugates. Chem Res Toxicol 18:1553-1562.

Venditti P, De Rosa R and Di Meo S (2003) Effect of thyroid state on $\mathrm{H}_{2} \mathrm{O}_{2}$ production by rat liver mitochondria. Mol Cell Endocrinol 205:185-192.

Associate Editor: Catarina Satie Takahashi 